\title{
Pre-transfer Editing of Serine Hydroxamate within the Active Site of Methanogenic-type Seryl-tRNA Synthetase ${ }^{\dagger}$
}

\author{
Ita Gruić-Sovulj, ${ }^{*}$ Morana Dulić, and Ivana Weygand-Đurašević \\ Department of Chemistry, Faculty of Science, University of Zagreb, Horvatovac 102a, 10000 Zagreb, Croatia
}

RECEIVED JANUARY 12, 2011; REVISED MARCH 11, 2011; ACCEPTED MARCH 17, 2011

\begin{abstract}
Aminoacyl-tRNA synthetases (aaRSs) maintain fidelity of protein synthesis by matching only cognate amino acid-tRNA pairs. Aminoacylation occurs through activation of amino acid to yield aminoacyl-adenylate followed by transfer of acyl-moiety to tRNA. Error-prone aaRSs achieve high level of accuracy using inherent hydrolytic activities towards noncognate aminoacyl-adenylate or misacylated tRNA (pre- and post-transfer editing).

Seryl-tRNA synthetases can be divided into two structurally different types: canonical and methanogenic-type. Both types have been shown to efficiently activate serine analogue serine hydroxamate (SerHX). Moreover, this analogue has been also eliminated by pre-transfer editing within the canonical synthetic site of yeast SerRS. Here we show that methanogenic-type SerRS from Methanosarcina barkeri clears misactivated SerHX similarly as the yeast enzyme: SerHX-adenylate is not expelled into solution, but is enzymatically hydrolyzed in a tRNA-independent manner. Since the enzyme lacks domain specialized in editing, this shows that methanogenic-type catalytic core is also capable to perform pre-transfer editing. (doi: 10.5562/cca1823)
\end{abstract}

Keywords: editing, proofreading, serine hydroxamate, seryl-tRNA synthetase

\section{INTRODUCTION}

Translation of the genetic code is the defining characteristic of all living systems. The first and most critical step in translation is the highly specific attachment of amino acids to their cognate tRNAs which is catalyzed by aminoacyl-tRNA synthetases (aaRSs). ${ }^{1,2}$ These enzymes establish the genetic code through the two-step aminoacylation reaction, which matches amino acids with nucleotide triplets encoded as anticodons in tRNAs. In the first step of aminoacylation known as activation, an aminoacyl-adenylate intermediate (aaAMP) is formed in an ATP-dependent manner. This intermediate remains noncovalently bound to the enzyme active site. In the second step known as aminoacyl transfer 2'- or 3'-OH group of the terminal adenosine of the tRNA attacks the carbonyl carbon of the mixed anhydride and transfer of aminoacyl moiety occurs.

AaRSs maintain fidelity of protein synthesis by pairing only cognate amino acid with cognate tRNA. However, given the similarity between amino acid substrates, some aaRSs cannot achieve high level of discrimination in the synthetic reaction alone. They proceed in activation and even transfer of noncognate ami- no acids to tRNA. ${ }^{3-5}$ As cell can tolerate only low level of mistake in protein biosynthesis (approximately 3 in 10000 (Ref. 6)), these aaRSs were forced to develop hydrolytic editing or proofreading. ${ }^{7}$ A mistake can be edited at the level of noncognate aa-AMP (pre-transfer editing) or at the level of misacylated tRNA (posttransfer editing) ${ }^{7}$. Cognate tRNA may stimulate pretransfer editing, but the exact mechanism by which tRNA stimulates hydrolysis of noncognate aa-AMP is not understood yet. Although majority of editing takes place through hydrolysis of noncognate aa-AMP or misacyl-tRNA, additional pathway comprising enhanced dissociation of noncognate aa-AMP from the enzyme and its subsequent non-enzymatic hydrolysis in solution may also occur. ${ }^{8,9}$ While pre-transfer editing was proposed to occur within the active synthetic site, ${ }^{10,11}$ post-transfer editing occurs inside the distant domain dedicated only to editing. ${ }^{7,12}$ Noncognate amino acid is brought to that distant active site by translocation of 3'-end of misacylated tRNA.

Seryl-tRNA synthetases (SerRS) covalently attach serine to cognate tRNA ${ }^{\text {Ser }}$. Beside canonical enzymes found in eukaryotes and prokaryotes, atypical SerRSs that are considerably different from the canonical en-

\footnotetext{
$\dagger$ This article belongs to the Special Issue Chemistry of Living Systems devoted to the intersection of chemistry with life.

* Author to whom correspondence should be addressed. (E-mail: gruic@chem.pmf.hr)
} 
zymes, were found in methanogenic archaea. Crystal structure of methanogenic-type SerRS from Methanosarcina barkeri (mMbSerRS) revealed two idiosyncratic features: a novel N-terminal tRNA-binding domain and a zinc ion in the active site. ${ }^{13}$ Additionally, a flexible region (serine ordering loop, SOL) within the active site, which undergoes notable induced fit rearrangement during serine binding, was recognized. The SOL peptide (17 amino acid long) changes its conformation drastically; from disordered conformation to arranged structure that comprises an $\alpha$-helix followed by an ordered loop.

Both SerRSs, from methanogen (atypical) and yeast (canonical) were shown to efficiently activate serine hydroxamate (SerHX) in an ATP-dependent manner to yield SerHX-AMP. ${ }^{14,15}$ This analogue of serine, having a hydroxamate group instead of carboxylate, has a potential as a microbial agent due to the observed inhibition of Escherichia coli growth primary through the inhibition of E. coli SerRS. ${ }^{16}$ Further inhibition studies performed on SerRSs from different organisms revealed that SerRSs from all domains of life are competitively inhibited by SerHX, albeit with different affinities. ${ }^{17}$ Although atypical (methanogenic-type) and canonical (e.g. from yeast) SerRSs both activate SerHX, the enzymes differ in further processing of SerHX during aminoacylation. While yeast SerRS does not transfer activated SerHX to the tRNA ${ }^{\mathrm{Ser}}$, mMbSerRS does. ${ }^{14}$ Yeast enzyme was also shown to clear misactivated SerHX in a tRNA-independent manner. To establish whether atypical enzyme is also able to edit SerHX, aminoacyl-adenylate synthesis assay has been employed. ${ }^{10,15,18}$ Here we show that analogously to the canonical enzyme, methanogenic-type SerRS hydrolyzes SerHX-AMP in a tRNA-independent manner. Substitutions of amino acid residues that participate in formation of the serine binding site (Trp396 and Gln400) also modulate the hydrolysis of aminoacyl-adenylate. This confirms that hydrolytic and synthetic reactions are intertwined within the synthetic site of methanogenictype enzyme.

\section{EXPERIMENTAL}

Protein Purification. The SerRS expression vector (pET15b-mMbSerRS ${ }^{19}$ ) coding for W396A mutant ${ }^{20}$ was used as a template for site-directed mutagenesis using a QuickChange mutagenesis kit (Stratagene). Introduction of second mutation (that results in W396A/Q400N substitution) was confirmed by DNA sequencing. Wild-type mMbSerRS and W396A/Q400N mutant were expressed in E. coli with addition of 1 mmol $\mathrm{dm}^{-3} \mathrm{ZnCl}_{2}$ to the media when the $\mathrm{OD}_{600}$ was approximately 0.1 , and purified employing affinity chromatography on Ni-NTA resin as reported previously. ${ }^{13}$ The concentration of proteins was determined by absorption at $280 \mathrm{~nm}$, using theoretical extinction coefficients. Determination of zinc content was done according to the previously published procedure, ${ }^{21}$ and both mMbSerRS wild-type and W396A/Q400N mutant contained approximately $2 \mathrm{~mol} \mathrm{Zn}^{2+}$ per mol of dimeric enzyme.

ATP-PP Exchange. ATP-PP $_{\mathrm{i}}$ exchange was measured at $37^{\circ} \mathrm{C}$ in $100 \mathrm{mmol} \mathrm{dm}^{-3} \mathrm{HEPES}$ pH 7.0, $20 \mathrm{mmol} \mathrm{dm}^{-3}$ $\mathrm{MgCl}_{2}, 25 \mathrm{mmol} \mathrm{dm}{ }^{-3} \mathrm{KCl}, 4 \mathrm{mmol} \mathrm{\textrm {dm } ^ { - 3 }}$ ATP and 1 $\mathrm{mmol} \mathrm{dm}{ }^{-3}\left[{ }^{32} \mathrm{P}\right] \mathrm{PP}_{\mathrm{i}}\left(0.002-0.01 \mathrm{mCi} \mathrm{cm} \mathrm{cm}^{-3}\right)$. Amino acid concentrations varied between 0.1 and $10 K_{\mathrm{m}}$. Due to too low activity of W396A/Q400N mutant in activation of D,L-SerHX, we were unable to determine $K_{\mathrm{m}}$ for this amino acid. The enzyme concentrations used were 100-500 nmol dm ${ }^{-3}$ for wild-type enzyme and $2 \mu \mathrm{mol}$ $\mathrm{dm}^{-3}$ for mMbSerRS W396A/Q400N mutant. The reactions were stopped with sodium acetate $\mathrm{pH} 5.0$ and SDS (final concentrations of $333 \mathrm{mmol} \mathrm{dm}^{-3}$ and of $0.067 \%$, respectively). The generated $\left[{ }^{32} \mathrm{P}\right] \mathrm{ATP}$ was separated from the remaining $\left.{ }^{32} \mathrm{P}\right] \mathrm{PP}_{\mathrm{i}}$ by thin layer chromatography (TLC) on polyethyleneimine plates (PEI), using 750 mmol dm ${ }^{-3} \mathrm{KH}_{2} \mathrm{PO}_{4} \mathrm{pH} 3.5$ and $4 \mathrm{~mol} \mathrm{dm}^{-3}$ urea as developer. ${ }^{22}$ The obtained signal was visualized on a Typhoon PhosphorImager and then quantified using ImageQuant software. Kinetic parameters were determined by fitting the data directly to the MichaelisMenten equation using non-linear regression. The parameters were obtained from at least three independent measurements. Each replicate was considered to be an independent data point.

\section{Aminoacyl-adenylate Synthesis Assay}

Aminoacyl-adenylate synthesis assay was done in $50 \mathrm{mmol} \mathrm{dm}^{-3}$ HEPES pH 7.6, $25 \mathrm{mmol} \mathrm{dm}^{-3} \mathrm{KCl}$, $20 \mathrm{mmol} \mathrm{dm}^{-3} \mathrm{MgCl}_{2}, 0.5 \mathrm{mmol} \mathrm{dm}{ }^{-3}$ ATP (0.01-0.1 $\left.\mathrm{mCi} \mathrm{cm}{ }^{-3}\right)$. D,L-SerHX was present in the concentration of $200 \mathrm{mmol} \mathrm{dm}^{-3}$. Concentration of serine was $1 \mathrm{mmol}$ $\mathrm{dm}^{-3}$ when the activity of the wild-type enzyme was studied, and $25 \mathrm{mmol} \mathrm{dm}$ when the editing of mMbSerRS W396A/Q400N variant was followed. In both cases concentration of serine was 20 -fold higher than $K_{\mathrm{m}}$ for serine measured in the ATP-PPi exchange assay. Both wild-type and mutant mMbSerRSs were 2-3 $\mu \mathrm{mol} \mathrm{dm}^{-3}$ and tRNA ${ }^{\text {Ser }}$ (when present) was $5-10 \mu \mathrm{mol} \mathrm{dm}{ }^{-3}$. The reaction was started by addition of amino acid or analogue and stopped by quenching with $400 \mathrm{mmol} \mathrm{dm}{ }^{-3}$ sodium acetate $(\mathrm{pH} 5.0) ; 0.1 \%$ SDS. Separation of $\alpha-\left[{ }^{32} \mathrm{P}\right]$-adenylate from aminoacyl$\alpha-\left[{ }^{32} \mathrm{P}\right]$-adenylate and $\alpha-\left[{ }^{32} \mathrm{P}\right]-\mathrm{ATP}$ was achieved by TLC followed by quantification and kinetic analysis as already described in. ${ }^{18}$ Background activity of nonenzymatic ATP hydrolysis was subtracted from velocities obtained in the presence of enzyme and amino acid or analogue. Control reaction containing $\left[{ }^{32} \mathrm{P}\right]-\mathrm{ATP}$ and SerRS showed that inherent ATPase activity of SerRS is close to the background activity. 
Table 1. Steady-state parameters for activation of serine and D,L-SerHX ${ }^{(a)}$

\begin{tabular}{lccc}
\hline & $K_{\mathrm{m}} / \mu \mathrm{mol} \mathrm{dm}$ & $\left(k_{\mathrm{cat}} / \mathrm{s}^{-1}\right.$ & \\
\hline wild-type $m M$ cat & & \\
Ser & & & 0.069 \\
$\mathrm{~m}$ & $/ \mathrm{s}^{-1} \mu \mathrm{mol}^{-1} \mathrm{dm}^{3}$ \\
D, L-SerHX & $44.9 \pm 7.7$ & $3.1 \pm 0.2$ & 0.00088 \\
mMbSerRS W396A/Q400N & $40.8 \pm 10.2$ & $(36 \pm 5) \times 10^{-3}$ & \\
Ser & & & 0.000074 \\
D, L-SerHX & $943 \pm 129$ & $(70 \pm 3) \times 10^{-3}$ & n.d. \\
\hline
\end{tabular}

The values represent the best fit value \pm s.e.m. of at least three independent experiments.

${ }^{\text {(a) All activities were measured by ATP-PP }} \mathrm{P}_{\mathrm{i}}$ exchange assay.

(b) From Ref. 14.

(c) $k_{\text {obs }}$ measured at saturating SerHX concentration.

n.d. not determined due to too low activity for reliable detection.

\section{RESULTS AND DISCUSSION}

\section{Methanogenic-type SerRS Possesses tRNA-indepen- dent Pre-transfer Editing}

We have recently shown that SerHX is efficiently activated by methanogenic-type SerRS (mMbSerRS, Table 1 (Ref. 14)). Activation proceeds with two orders of magnitude lower $k_{\text {cat }}$ compared to activation of cognate serine. In contrast, $K_{\mathrm{m}}$ values for SerHX and serine were very similar. Obtained discrimination factor suggests that mMbSerRS needs editing activity to cope with its incapability to eliminate SerHX in the synthetic reaction alone. Similar situation was observed with yeast and $E$. coli SerRSs, and editing of SerHX was directly demonstrated for both enzymes. ${ }^{15}$ To investigate whether mMbSerRS also eliminates activated SerHX, aminoacyl-adenylate synthesis assay has been performed. ${ }^{18}$ This assay allows us to directly follow the fate of aa$\left[{ }^{32} \mathrm{P}\right]$-AMP and its hydrolytic product $\left[{ }^{32} \mathrm{P}\right]$-AMP. Using this assay we observe that mMbSerRS produces a significant amount of AMP in the presence of SerHX. A small amount of SerHX-AMP was also observed (Fig-

(a)

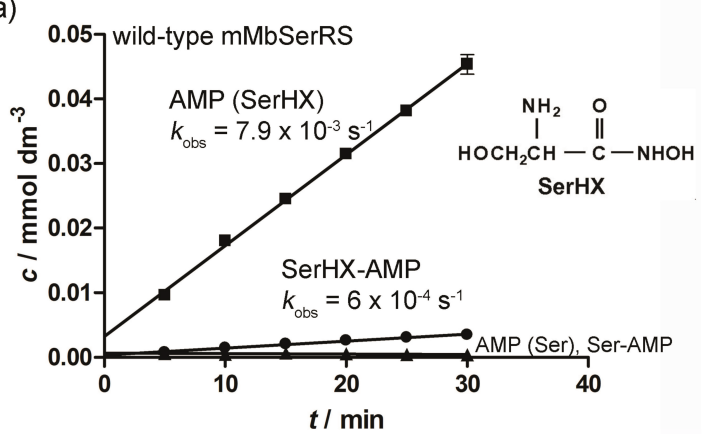

ure 1a). In contrast, in the presence of cognate serine, mMbSerRS does not stimulate Ser-AMP and/or AMP production over their stoichiometric amounts with respect to the used enzyme concentration (Figure 1a). Observed catalytic constant $\left(k_{\text {obs }}\right)$ for AMP formation under the saturating conditions of amino acid or analogue, was 20-fold higher in the presence of SerHX than serine (Table 2). Stimulation of AMP formation in the presence of noncognate amino acid is generally taken as diagnostic of hydrolytic editing because enhanced consumption of ATP should parallel activation/hydrolysis cycles utilized by the enzyme confronted with noncognate amino acid. Thus, presented data revealed that mMbSerRS employs editing to clear misactivated SerHX. However, pre-transfer editing may occur through enhanced dissociation of aa-AMP from the enzyme followed by its subsequent non-enzymatic hydrolysis in solution or through enzymatic hydrolysis of aa-AMP. To investigate whether mMbSeRS edits SerHX-AMP through enhanced dissociation or through enzyme based hydrolytic activity, the rate of nonenzymatic hydrolysis of SerHX-AMP was measured

(b)

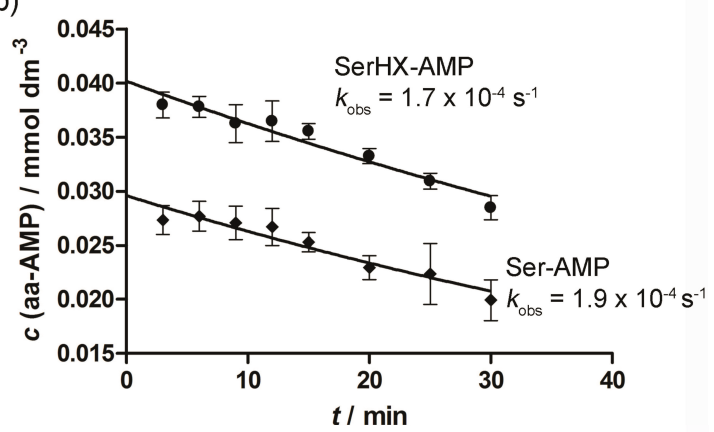

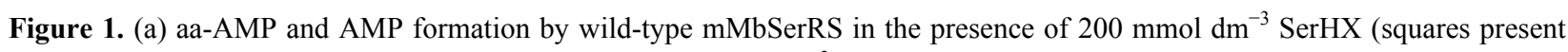
AMP and circles denote SerHX-AMP formation) or $1 \mathrm{mmol} \mathrm{dm}^{-3}$ Ser (for clarity, triangles present both AMP and Ser-AMP formation). (b) Non-enzymatic hydrolysis of SerHX-AMP (squares) and Ser-AMP (rhombs) measured in solution. All data are measured in at least three independent experiments. Standard errors are represented by error bars. 
Table 2. Observed steady-state catalytic constants for AMP formation $^{(\text {a) }}$

\begin{tabular}{lc}
\hline & $k_{\mathrm{obs}} / \mathrm{s}^{-1}$ \\
\hline wild-type mMbSerRS & $(0.36 \pm 0.03) \times 10^{-3} \mathrm{~s}^{-1}$ \\
Ser & $(7.9 \pm 0.6) \times 10^{-3} \mathrm{~s}^{-1}$ \\
SerHX & \\
mMbSerRS W396A/Q400N & $(3.7 \pm 0.4) \times 10^{-3} \mathrm{~s}^{-1}$ \\
Ser & $(1.1 \pm 0.1) \times 10^{-3} \mathrm{~s}^{-1}$ \\
SerHX
\end{tabular}

The values represent the mean \pm s.d. of at least three independent experiments.

(a) All activities were measured by aminoacyl-adenylate synthesis assay in the absence of tRNA.

and compared to the rate of AMP formation. Nonenzymatic aa-AMP hydrolysis was followed by our previously developed cold chase assay, ${ }^{18}$ where enzyme is mixed with amino acid and $\alpha-\left[{ }^{32} \mathrm{P}\right]-\mathrm{ATP}$ in order to accumulate aa- $\left[{ }^{32} \mathrm{P}\right]-A M P$. The reaction is then quenched with 10- to 100-fold molar excess with respect to $\left[{ }^{32} \mathrm{P}\right]$-ATP and the conversion of aa-AMP to AMP monitored. Observed rate of non-enzymatic SerHX-AMP hydrolysis was $1.7 \times 10^{-4} \mathrm{~s}^{-1}$ (Figure 1b), 40-fold lower than the observed rate of AMP formation by mMbSerRS. As AMP is formed significantly faster in the presence of enzyme this confirms that methanogenic-type SerRS possesses hydrolytic pre-transfer editing of SerHX. It is known that cognate tRNA may stimulate noncognate aa-AMP hydrolysis. However, we could not detect enhancement of the SerHX editing in the presence of $\mathrm{tRNA}^{\mathrm{Ser}}$ (data not shown). Recently solved crystal structure of mMbSerRS revealed that this enzyme is built from two domains; a catalytic domain that features $\mathrm{Zn}$-mediated amino acid activation and the tRNA binding domain. An additional domain that may have been involved in hydrolytic editing was not observed. $^{13}$ This strongly suggests that hydrolysis of SerHX-AMP occurs within the synthetic site as was previously proposed for both yeast and E. coli enzymes. ${ }^{15}$ Presented data show that canonical and atypical SerRS synthetic sites although differ in the mechanism of amino acid recognition and its metal dependency, share a feature of tRNA-independent pre-transfer editing. As discussed previously, ${ }^{13}$ similarities between methanogenic-type SerRS, ThrRS and bacterial-type ProRS may support a concept of the ancient origin of methanogenic-type SerRS suggesting that Zn-dependent amino acid recognition predates segregation of SerRS and ThrRS into enzymes with distinct specificities. Thus, the observation of editing by an ancient mMbSerRS additionally supports an idea that primordial catalytic module of aaRS had the ability to hydrolyze aa-AMP in a tRNA-independent manner. The presence of this activity as a relic from primordial times has been documented in many contemporary aaRSs like ThrRS, ${ }^{11}$ ProRS, ${ }^{9,23}$ LeuRS, ${ }^{5,24,25,26}$ IleRS, ${ }^{8,10}$ ValRS. ${ }^{10,27}$ This tempted us to hypothesize that hydrolytic editing is an inherent feature of aaRSs synthetic sites that evolved in parallel with the amino acid activation.

Weak misactivation of threonine was also observed by both yeast and mMbSerRSs, but discrimination factors did not strongly support necessities for editing of the threonine during protein synthesis. ${ }^{13,15}$ In a separately tested editing assay (aminoacyl-adenylate synthesis assay, ${ }^{18}$ ), yeast enzyme was shown to stimulate AMP formation in the presence of noncognate threonine. ${ }^{15}$ For methanogenic-type enzyme no preferential stimulation of AMP in the presence of threonine compared to the cognate serine has been observed. Interestingly both enzymes activate SerHX more efficiently than threonine. ${ }^{13,14,15}$ Structural and solution-based studies ${ }^{28}$ pointed out that the covalent bond is established between hydroxyl oxygen of SerHX and $\alpha$-phosphate of ATP. This is in agreement with a predicted higher nucleophilicity of the oxygen atom compared to the nitrogen atom of the hydroxylamine moiety of SerHX. Thus, it seems that extra bond length in SerHX-AMP intermediate is tolerated by both yeast and methanogenic-type SerRSs. Particularly interesting finding is that although not a metabolite that is naturally occurring in the cell, SerHX is nevertheless edited by both enzymes. The presence of the additional bond may induce slight perturbation within the synthetic site and thus expose this region to a water nucleophile. Alternatively, it is possible that the nitrogen atom stimulates the hydrolytic activity either by acting as a base that may enhance water nucleophilicity or as hydrogen-bonding partner that may influence positioning of a water molecule within the synthetic site.

\section{Active site mutations that modulate pre-transfer editing activity of $\mathbf{m M b S e r R S}$}

An increasing number of evidence supports a model wherein pre-transfer editing occurs within the synthetic site. $^{9-11,15,23-26}$ The model was built on the observed pretransfer editing activity of aaRS mutants having editing domain either inactivated or completely eliminated. To get better insight into location of hydrolytic activity toward SerHX-AMP within the synthetic site, we have substituted two synthetic site residues Trp396 and Gln400 (Figure 2). Trp396 packs against serine amino group acting as a gatekeeper that plays an important role in determining the size of the amino-acid binding pocket. On the other hand, Gln400 contacts carbonyl oxygen of the serine substrate via direct hydrogen bond. ${ }^{13}$ The rationale was to substitute the $G \ln 400$, a residue that may participate in positioning of catalytic water due to its location in the close vicinity of the scissile bond. In 


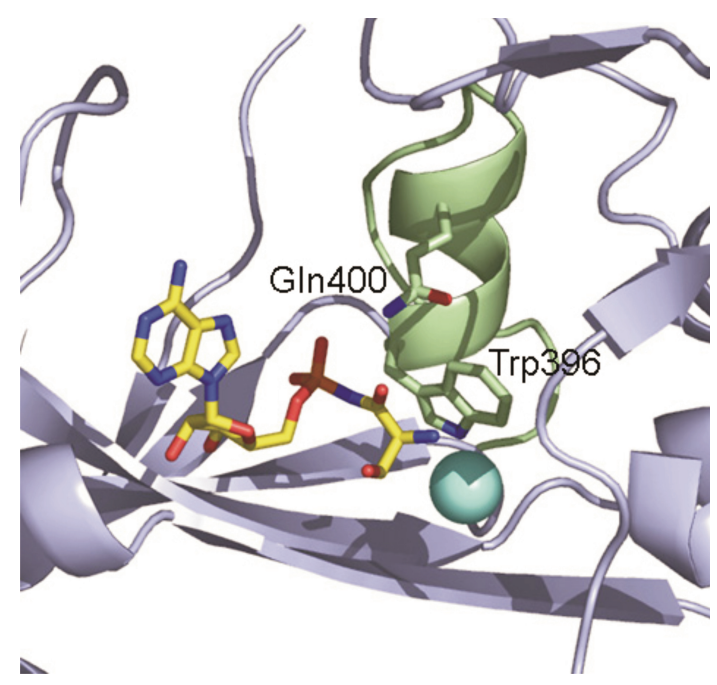

Figure 2. Crystal structure of the mMbSerRS active site occupied with seryl-adenylate analogue. ${ }^{13}$ SOL peptide is colored in green, zinc atom is shown in cyan, and Ser-AMS in yellow. Trp396 and Gln400 are shown as sticks.

addition, W396A substitution was introduced to broaden the active site and stimulate the entrance of catalytic water. Thus, mMbSerRS W396A/Q400N mutant was produced and tested in activation and editing of serine and SerHX (Tables 1 and 2).

Unexpectedly, introduced mutations severely decreased enzyme's ability to activate serine (Table 1 ). The $K_{\mathrm{m}}$ was 20 -fold higher and the $k_{\text {cat }} 40$-fold lower than for wild-type mMbSerRS. The notable drop in $k_{\text {cat }}$ was surprising since W396A substitution was previously reported as the one that primarily modulate $K_{\mathrm{m}}$ (Ref. 20) while substitution of Gln400 with Ala did not significantly influence any parameter of the steady-state activation of serine. ${ }^{29}$ Both residues belong to a small peptide (SOL) that becomes ordered during binding of serine (Figure 2). ${ }^{13}$ Ordering of SOL participates in formation of the serine binding site, with Trp396 playing a prominent role in positioning of the peptide within the active site. ${ }^{29}$ Data presented here unveil that Gln400 may also participate in positioning of SOL particularly when Trp396 is missing. Once both residues are substituted, as in mMbSerRS W396A/Q400N mutant, the SOL is mispositioned within the active site leading to the severely impaired activation (Table 1). Activation of SerHX highly parallels activation of serine (Table 1). However, due to the observed low activity of mMbSerRS W396A/Q400N variant in activation of SerHX, we could not extract steady-state parameters from our kinetic analysis. The measurable activity was achieved only at saturating SerHX concentrations, allowing us to determine only $k_{\mathrm{obs}}$, the best representative of the $k_{\text {cat }}$.

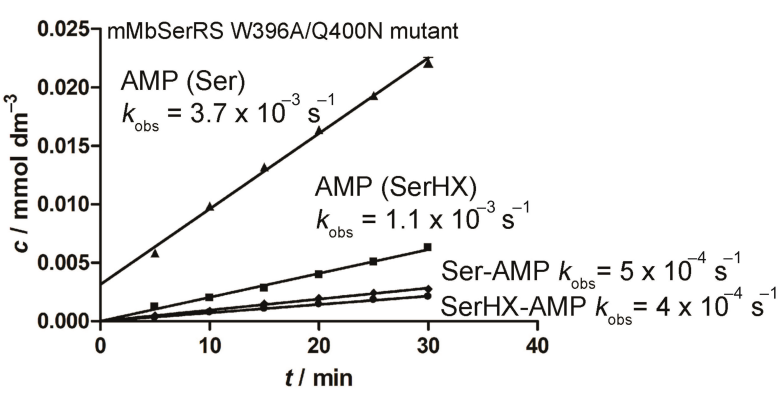

Figure 3. aa-AMP and AMP formation by mMbSerRS W396A/Q400N mutant. Formation of AMP in the presence of serine $\left(25 \mathrm{mmol} \mathrm{dm}{ }^{-3}\right)$ is presented by triangles and in the

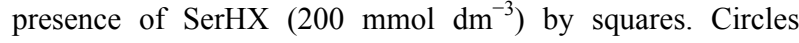
denote SerHX-AMP formation and rhombs Ser-AMP formation. All data are measured in at least three independent experiments. Standard errors are represented by error bars.

To address influence of W396A and Q400N substitutions on pre-transfer editing, aminoacyl-adenylate synthesis assay with mMbSerRS W396A/Q400N mutant was performed. Observed catalytic constant for AMP formation in the presence of SerHX was 5-fold lower than the catalytic constant obtained with the wildtype enzyme (Table 2), but still 5-fold higher than the rate of non-enzymatic SerHX-AMP hydrolysis (Figure 1a). In addition, SerHX-AMP still accumulates with slower rate than AMP (Figure 3). All together, this strongly suggests that mutated active site hydrolyzes SerHX-AMP. The observable rate of hydrolysis may be limited by slow rate of SerHX activation observed for mMbSerRS W396A/Q400N mutant. Therefore it is uncertain to assign 5-fold decrease in AMP formation exclusively to the impairment of the pre-transfer editing.

Unexpectedly, mMbSerRS W396A/Q400N mutant displayed significantly enhanced AMP formation in the presence of cognate serine (Figure 3). Observed catalytic constant obtained by the mutant was 10 -fold higher than the one obtained by the wild-type enzyme (Table 2). Using the same arguments as before (relatively fast rate of AMP formation compared to the rate of non-enzymatic hydrolysis, in this case of Ser-AMP $\left(0.19 \times 10^{-4} \mathrm{~s}^{-1}\right.$, Figure $\left.1 \mathrm{~b}\right)$ and slower accumulation of Ser-AMP compared to AMP) we can conclude that W396A/Q400N mutant hydrolyzes Ser-AMP within the synthetic site. Interestingly, broadening of the synthetic site did not enhance dissociation of Ser-AMP or SerHXAMP from the enzyme, but rather modulates their rates of hydrolysis within the synthetic site. Obviously, the stability of mMbSerRS:aa-AMP complex has not been significantly perturbed by the introduced substitutions, suggesting that majority of interactions occur with AMP portion of the aa-AMP molecule.

Presented data show that the synthetic site of atypical SerRS performs pre-transfer editing in a similar 
way as canonical SerRS synthetic site does. Mutational analysis revealed that activation (synthetic reaction) and pre-transfer editing (hydrolytic reaction) are deeply intertwined, presumably as a consequence of highly overlapping sites of occurrence. Residues like Trp396 and $\mathrm{G} \ln 400$ that participate in the formation of the serine binding site also influence the hydrolytic activity toward aa-AMP. This makes assignment of the amino acid residues involved in the hydrolytic reaction a very difficult task. Perturbation of one activity may modulate efficiency or even specificity of the other. Interconnection of synthetic and editing reactions within the same synthetic site may have constrained evolution of pretransfer editing. Therefore, to attain the protein synthesis within the tolerable error, while keeping the efficiency of the amino acid activation at high level, more promiscuous aaRSs were forced to acquire additional active site dedicated only for hydrolytic editing. This site located at the remote editing domain becomes reachable through the conformational flexibility of the tRNA that allows its single stranded 3'-end to access both synthetic and editing site.

Acknowledgements. We thank Silvija Bilokapić for constructive discussions. This work was supported by grant from the Ministry of Science, Education and Sports of the Republic of Croatia (119-0982913-1358).

\section{REFERENCES}

1. M. Ibba and D. Soll, Annu. Rev. Biochem. 69 (2000) 617-650.

2. M. Ibba and D. Soll, EMBO Rep. 2 (2001) 382-387.

3. A. R. Fersht, Biochemistry 16 (1977) 1025-1030.

4. A. R. Fersht and M. M. Kaethner, Biochemistry 15 (1976) 3342-3346.

5. S. Englisch, U. Englisch, F. von der Haar, and F. Cramer, Nucleic Acids Res 14 (1986) 7529-7539.

6. R. B. Loftfield and D. Vanderjagt, Biochem. J. 128 (1972) 13531356.

7. J. Ling, N. Reynolds, and M. Ibba, Annu Rev Microbiol 63
(2009) 61-78.

8. H. Jakubowski and A. R. Fersht, Nucleic Acids Res 9 (1981) 3105-3117.

9. S. Hati, B. Ziervogel, J. Sternjohn, F. C. Wong, M. C. Nagan, A. E. Rosen, P. G. Siliciano, J. W. Chihade, and K. Musier-Forsyth, J. Biol. Chem. 281 (2006) 27862-27872.

10. M. Dulić, N. Cvetešić, J. J. Perona, and I. Gruić-Sovulj, J. Biol.Chem. 285 (2010) 23799-23809.

11. A. Minajigi and C. S. Francklyn, J. Biol.Chem. 285 (2010) 23810-23817.

12. L. F. Silvian, J. Wang, and T. A. Steitz, Science 285 (1999), 1074-1077.

13. S. Bilokapić, T. Maier, D. Ahel, I. Gruić-Sovulj, D. Soll, I. Weygand-Durašević, and N. Ban, EMBO J. 25 (2006) 2498-2509.

14. I. Gruić-Sovulj, M. Dulić, J. Jaric, N. Cvetešić, K. Majsec, and I. Weygand-Durašević, Croat. Chem. Acta 83 (2010) 163-169.

15. I. Gruić-Sovulj, J. Rokov-Plavec, and I. Weygand-Đurašević, FEBS Lett. 581 (2007) 5110-5114.

16. T. Tosa and L. I. Pizer, J. Bacteriol. 106 (1971) 966-971.

17. D. Ahel, D. Slade, M. Mocibob, D. Soll, and I. WeygandDurašević, FEBS Lett. 579 (2005) 4344-4348.

18. I. Gruić-Sovulj, N. Uter, T. Bullock, and J. J. Perona, J. Biol. Chem. 280 (2005) 23978-23986.

19. D. Korenčić, C. Polycarpo, I. Weygand-Durašević, and D. Soll, J. Biol. Chem. 279 (2004) 48780-48786.

20. S. Bilokapić, J. Rokov Plavec, N. Ban, and I. WeygandDurašević, FEBS J. 275 (2008) 2831-2844.

21. J. A. Landro and P. Schimmel, Proc. Natl. Acad. Sci. USA 90 (1993) 2261-2265.

22. N. T. Uter, I. Gruić-Sovulj, and J. J.Perona, J. Biol. Chem. 280 (2005) 23966-23977.

23. K. E. Splan, M. E. Ignatov, and K. Musier-Forsyth, J. Biol. Chem. 283 (2008) 7128-7134.

24. M. T. Boniecki, M. T. Vu, A. K. Betha, and S. A. Martinis, Proc Natl Acad Sci USA 105 (2008) 19223-19228.

25. B. Zhu, P. Yao, M. Tan, G. Eriani, and E. D. Wang, J. Biol. Chem. 284 (2009) 3418-3424.

26. M. Tan, B. Zhu, X. L. Zhou, R. He, X. Chen, G. Eriani, and E. D. Wang, J. Biol. Chem. 285 (2010) 3235-3244.

27. H. Jakubowski, Biochemistry 19 (1980) 5071-5078.

28. H. Belrhali, A. Yaremchuk, M. Tukalo, K. Larsen, C. BerthetColominas, R. Leberman, B. Beijer, B. Sproat, J. Als-Nielsen, G. Grübel, J.-F. Legrand, M. Lehmann, and S. Cusack, Science $\mathbf{2 6 3}$ (1994) 1432-1436.

29. M. Dulić, J. Požar, S. Bilokapić, I. Weygand-Đurašević, and I. Gruić-Sovulj, Biochimie 93 (2011). 